\title{
Minimizing the Bright/Shadow Focal Spot Size with Controlled Side-Lobe Increase in High-Numerical-Aperture Focusing Systems
}

\author{
S. N. Khonina and S. G. Volotovskiy \\ Image Processing Systems Institute RAS, Molodogvardeiskaya Street 151, Samara 443001, Russia \\ Correspondence should be addressed to S. N. Khonina; khonina@smr.ru
}

Received 8 July 2013; Accepted 13 September 2013

Academic Editor: Augusto Beléndez

Copyright (C) 2013 S. N. Khonina and S. G. Volotovskiy. This is an open access article distributed under the Creative Commons Attribution License, which permits unrestricted use, distribution, and reproduction in any medium, provided the original work is properly cited.

\begin{abstract}
Minimizing the bright/shadow focal spot size for differently polarized incident waves through the additional apodization of the focusing system output pupil by use of an optical element with the vortex phase dependence on angle and the polynomial amplitude dependence on radius is studied. The coefficients of the radial polynomial were optimized with the aim of fulfilling certain conditions such as the energy efficiency preservation and keeping the side lobes under control. The coefficients were chosen so as to minimize the functional using Brent's method.
\end{abstract}

\section{Introduction}

Recent years have seen the publication of a large number of articles dealing with obtaining a smaller transverse size of the focal spot using a high NA focusing system [1-5]. The smallest transverse size of the focal spot has been obtained for a radially polarized beam in which the longitudinal component makes the maximal contribution to the total intensity.

There is a variety of techniques to gain the contribution of the longitudinal component to the total intensity, including the use of an annular aperture which allows only the peripheral part of radiation to pass through the lens $[1,2]$ and the use of additional phase optical elements, which provides a higher energy efficiency [3-5].

However, this study is concerned with not simply controlling the contribution of different electric field components into the focal spot region [5], but rather minimizing the (bright/dark) focal spot size by optimally selecting the pupil's transmission function.

In [6], it was proposed that the focal spot formed by a high NA focusing system should be reduced in size using radially polarized Laguerre-Gauss modes of higher radial order, devoid of vortex phase component.
The positive role of the vortex phase function which allows obtaining a smaller focal spot in individual components of the sharply focused electric field at different polarization types was analyzed in [4], whereas the possibility of obtaining a smaller focal spot in terms of total intensity by introducing additional variations of radius was shown in [5]. The use of the transmission functions in the form of Zernike polynomials, including those containing vortex phase dependence, was considered in [7]. Thus, it has become possible to simultaneously introduce vortex phase dependence and amplitude variations on radius. Note that it has also been shown that it is possible to reduce not only the bright spot but the shadow region as well.

In areas such as optical trapping and micromanipulation [8], STED-microscopy [9], and shadow microscopy [10], there has also been a problem of focusing into a shadow spot or a bright ring with a zero intensity region tightly localized at the center. The described focal distribution is implemented through introducing the phase singularity into the beam under focusing.

In this work, we look into minimizing the bright/shadow focal spot size for differently polarized beams through the additional apodization of the focusing system using an 
optical element with the vortex phase dependence on angle and the polynomial amplitude dependence on radius. The order and direction of the phase vortex component were chosen with regard to the polarization of the focusing system incident beam [11], whereas the coefficients of the low-degree polynomial were optimized so as to fulfill certain conditions, in particular, the energy efficiency preservation and keeping the side lobes at a desired level. The coefficients were chosen so as to minimize the functional using Brent's method [12].

\section{Scalar Diffraction Limit}

In the scalar case, the result of focusing a bounded plane wave of wavelength $\lambda$ and wavenumber $k=2 \pi / \lambda$ with a lens of radius $R$ and focal length $f$ is given by the complex amplitude as follows:

$$
\begin{aligned}
E_{0}(\rho, \varphi) & =\frac{k}{i f} \exp (i k f) \int_{0}^{R} J_{0}\left(\frac{k r \rho}{f}\right) r \mathrm{~d} r \\
& =\exp (i k f) \frac{R}{i \rho} J_{1}\left(\frac{k \rho R}{f}\right),
\end{aligned}
$$

where $(\rho, \varphi)$ are the polar coordinates in the focal plane and $J_{n}(x)$ is the Bessel function of the $n$th order. Note that the integral (1) is calculated precisely.

The focal spot radius can be derived from the first zero of the first-order Bessel function as follows:

$$
\rho_{l}=\frac{3.83 f}{k R} \approx \frac{0.61 \lambda}{\mathrm{NA}}
$$

where NA is the lens numerical aperture.

A widely known approach to obtaining a tighter focal spot is by replacing the full aperture by a narrow peripheral ring of width $\delta$. In this case, the resulting spectrum is associated with the zero-order Bessel function (for small $\delta$, the integrand can be calculated at midpoint) as follows:

$$
\begin{aligned}
E_{0}(\rho, \varphi) & =\frac{k}{i f} \exp (i k f) \int_{R-\delta}^{R} J_{0}\left(\frac{k r \rho}{f}\right) r \mathrm{~d} r \\
& \approx \exp (i k f) \frac{k R \delta}{i f} J_{0}\left(\frac{k \rho R}{f}\right),
\end{aligned}
$$

with the focal spot radius derived from the first zero of the zero-order Bessel function as follows:

$$
\rho_{l \delta}=\frac{2.4 f}{k R} \approx \frac{0.38 \lambda}{\mathrm{NA}} \text {. }
$$

The comparison between (1) and (3) suggests that the smaller transverse size of the focal spot is achieved at the cost of lower focal spot intensity $(\rho=0)$. At $\delta \sim \lambda$, the focal spot intensity will drop in direct proportion to the focal length squared.

Thus, with the aberration-free full-aperture focusing system $(\mathrm{NA}=1)$, the scalar diffraction-limited radius of the focal spot is $\rho_{l} \approx 0.61 \lambda$ (intensity $\mathrm{FWHM}_{l} \approx 0.51 \lambda$ ), whereas with a narrow annular aperture, it is $\rho_{l \delta} \approx 0.38 \lambda\left(\mathrm{FWHM}_{l \delta} \approx\right.$ $0.36 \lambda)$.
Also of great interest is the problem of focusing into a shadow spot or a bright ring with the zero intensity tightly localized at the center. The described focal pattern can be generated by introducing a vortex phase singularity into the beam under focusing.

In the scalar case, the result of focusing of a bounded plane wave with the first-order vortex phase singularity is given by

$$
E_{1}(\rho, \varphi)=\frac{k}{i f} \exp (i k f) \exp (i \varphi) \int_{0}^{R} J_{1}\left(\frac{k r \rho}{f}\right) r \mathrm{~d} r .
$$

The integral in (5) is normally expressed through a superposition of the Bessel and Struve functions [13] in the form

$$
\int_{0}^{R} J_{1}(\alpha r) r \mathrm{~d} r=\frac{\pi R}{2 \alpha}\left[J_{1}(\alpha R) H_{0}(\alpha R)-J_{0}(\alpha R) H_{1}(\alpha R)\right],
$$

which is inconvenient to analyze. Because of this, the integral in (5) can be expressed through a hypergeometric function [13] as follows:

$$
\int_{0}^{R} J_{1}(\alpha r) r \mathrm{~d} r=\frac{\alpha R^{3}}{6}{ }_{1} F_{2}\left(\frac{3}{2} ; \frac{5}{2}, 2 ;-\frac{\alpha^{2} R^{2}}{4}\right),
$$

where ${ }_{1} F_{2}\left(x ; y_{1}, y_{2} ; z\right)=\sum_{n=0}^{\infty}(x)_{n} /\left(\left(y_{1}\right)_{n} \cdot\left(y_{2}\right)_{n}\right) \cdot\left(z^{n} / n !\right)$, $(a)_{0}=1,(a)_{n}=a \cdot(a+1) \cdots(a+n-1)$.

Thus, (5) can be written as

$$
\begin{aligned}
E_{1}(\rho, \varphi)= & \exp (i k f) \exp (i \varphi) \\
& \times \frac{k^{2} R^{3} \rho}{6 i f^{2}}{ }_{1} F_{2}\left(\frac{3}{2} ; \frac{5}{2}, 2 ;-\frac{(k \rho R)^{2}}{4 f^{2}}\right) .
\end{aligned}
$$

By retaining in (8) only the first two terms and equating the derivative to zero, the estimate of the focal ring radius is given by

$$
\widehat{\rho}_{d}=\frac{2 \sqrt{10} f}{3 R k} \approx \frac{0.336 \lambda}{\mathrm{NA}} .
$$

The calculation of the hypergeometric function (e.g., using Matlab) or the calculation of the integral in (8) gives a larger value of radius (Figure 1, dashed line) as follows:

$$
\rho_{d}=\frac{0.39 \lambda}{\mathrm{NA}}
$$

The focal spot size can also be made smaller by applying a narrow annular diaphragm allowing only the peripheral rays to be transmitted as follows:

$$
\begin{aligned}
E_{1}(\rho, \varphi) & =\frac{k}{i f} \exp (i k f) \exp (i \varphi) \int_{R-\delta}^{R} J_{1}\left(\frac{k r \rho}{f}\right) r \mathrm{~d} r \\
& \approx \exp (i k f) \exp (i \varphi) \frac{k R \delta}{i f} J_{1}\left(\frac{k \rho R}{f}\right) .
\end{aligned}
$$




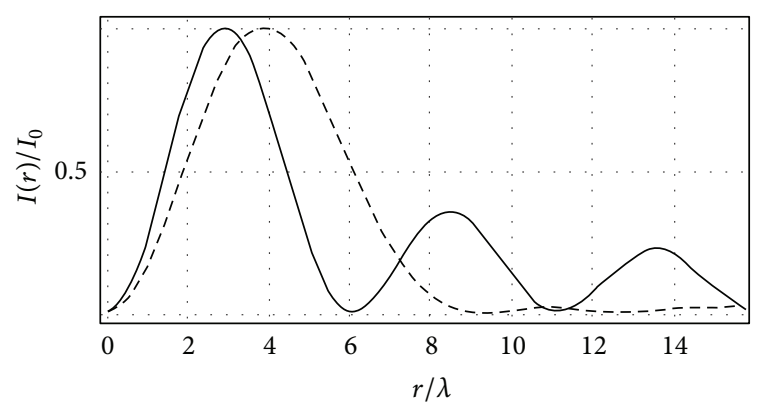

FIGURE 1: The plots of the normalized intensity distribution analytically calculated for (8) (dashed line) and (11) (solid line) at NA $=0.1$.

In this case, the light ring radius is derived from the maximal value of the first-order Bessel function (Figure 1, solid line) as follows:

$$
\rho_{d \delta}=\frac{1.84 f}{k R} \approx \frac{0.293 \lambda}{\mathrm{NA}} .
$$

The smaller size is attained at the sacrifice of the energy focused in the central ring (intensity is proportional to $\delta^{2}$ ) and, hence, a lower intensity of the light barrier. Note, however, that the compactness of the light vortex may prove to be a more important parameter than its height.

The above-described computations suggest that, with the aberration-free full-aperture focusing system $(\mathrm{NA}=1)$, the scalar diffraction-limited radius of the light ring is $\rho_{d} \approx$ $0.39 \lambda$ (with the ring's internal radius defined by the same value at intensity FWHM), whereas, with the narrow annular aperture, we have $\rho_{d \delta} \approx 0.29 \lambda$.

Thus, the diffraction-limited radius of the light ring is smaller than that of the light spot [14].

With high NA focusing systems, the achievement of the diffraction-limited size is problematic because different electric field components contribute to the focal region $[4,5]$. This problem is addressed with the use of specially polarized beams, thus enabling the contribution of an individual component to be maximized. For example, with a radially polarized incident beam, the diffraction-limited focal spot size can be achieved with the aid of a narrow annular aperture $[1,2]$ or using full-aperture transmission functions characterized by higher energy efficiency [3-7, 11, 14-16]. The full-aperture apodization can be most easily implemented by complementing the focusing system with phase optical elements $[3-5,14-16]$ and focusing higher-order laser modes $[6,7]$. In the latter case, the intensity distribution at the system's output pupil can be represented as a polynomial. Below, we discuss the possibility of minimizing the transverse focal spot with the aid of low-order polynomials under the fulfillment of certain conditions, including the energy efficiency preservation and keeping the side-lobe level under control.

\section{Model of a High NA Focusing System in Debye's Approximation}

For a high NA focusing system, the near-focus vector electric field in a homogeneous dielectric medium can be described in Debye's approximation [17], which holds if the focal length of the optical system is much larger than the incident wavelength as follows:

$$
\begin{aligned}
& \mathbf{E}(\rho, \varphi, z) \\
& =-\frac{i f}{\lambda} \int_{0}^{\Theta} \int_{0}^{2 \pi} B(\theta, \phi) T(\theta) \mathbf{P}(\theta, \phi) \\
& \quad \times \exp [i k(\rho \sin \theta \cos (\phi-\varphi)+z \cos \theta)] \\
& \quad \times \sin \theta \mathrm{d} \theta \mathrm{d} \phi,
\end{aligned}
$$

where $(\rho, \varphi, z)$ are the cylindrical coordinates in the focal region, $(\theta, \phi)$ are the spherical angular coordinates of the focusing system's output pupil, $\Theta$ is the maximal value of the azimuthal angle related to the system's NA, $B(\theta, \phi)$ is the transmission function, $T(\theta)=\sqrt{\cos \theta}$ is the pupil's apodization function for aplanatic systems, $\mathbf{P}(\theta, \phi)$ is the polarization matrix, and $f$ is the focal length.

Below, we analyze sharply focusing differently polarized laser beams with the transmission function $B(\theta, \phi)$ defined by the following superposition:

$$
B_{m}(\theta, \phi)=\exp (i m \phi) \sum_{s=0}^{S} c_{s} \sin ^{s} \theta
$$

where $c_{s}$ are the polynomial coefficients at a fixed value of vortex order $m$.

With the above-defined incident beam, (13) can be essentially simplified for the widely used types of polarization.

Then, the vector electric field in the focal plane can be calculated using a $1 \mathrm{D}$ integral [4] as follows:

$$
\mathbf{E}_{m}(\rho, \varphi, z)=-i k f \int_{0}^{\Theta} \mathbf{Q}_{m}(\rho, \varphi, \theta) q(\theta) \mathrm{d} \theta
$$

where $q(\theta)=\exp (i k z \cos \theta) \sqrt{\cos \theta} \sum_{s=0}^{S} c_{s} \sin ^{s+1} \theta$, with the form of $\mathbf{Q}_{m}(\rho, \varphi, \theta)$ depending on the input field polarization.

\section{Optimization of the Transmission Function of the Focusing System}

After choosing a definite type of polarization and the phase vortex order $m$, the coefficients in (14) were optimized through minimizing a functional relating the output distribution intensity in (15) and a target function $T(\rho, \varphi)$ in the focusing region as follows:

$$
\Phi\left\{\left|\mathbf{E}_{m}(\rho, \varphi, z=0)\right|^{2} ; T(\rho, \varphi)\right\} \underset{c_{s}}{\longrightarrow} \min .
$$

The target function is a superposition of functions that define the conditions which the focal intensity distribution 
needs to satisfy. In particular, these conditions require that the intensity be localized in a definite region or imply a penalty if the normalized intensity becomes higher than a threshold on a certain interval.

For a fixed polar angle $\varphi$, the constituent functions of the functional (16) will only depend on the radius, making it possible to visually illustrate in which way the above-described conditions are imposed on the intensity distribution in (15), as seen in Figure 2. To achieve a definite trade-off between the minimal focal spot size and suppressed side lobes, it is required to impose the condition that the energy should be localized in a focal plane's circle of radius $\rho_{\varepsilon}$ and provide a definite level of side lobes, $\mu$, in a ring of radii $\left[\rho_{\varepsilon}, \rho_{\Delta}\right]$. In the region of radius larger than $\rho_{\Delta}$, the intensity distribution is not controlled.

The functional (15) was minimized at a fixed value of the polar angle $\varphi$ using Brent's method [12]. The method was implemented using the public-domain software from ALGLIB [18].

\section{Radial Polarization}

5.1. Minimizing the Focal Spot. It has been known $[1-4,6]$ that the smallest transverse size of the focal spot is provided by a radially polarized incident beam, as in this case the contribution of the longitudinal component to the total intensity on the optical axis is maximal.

For the radially polarized wave, the field in (15), which depends on the laser beam's vortex component $\exp (\operatorname{im} \phi)$, takes the (Cartesian) form,

$$
\begin{aligned}
& \mathbf{E}_{m}(\rho, \varphi, z) \\
& =-i^{m+1} k f \exp (i m \varphi) \\
& \times \int_{0}^{\Theta}\left[\begin{array}{l}
\frac{i}{2}\left[e^{i \varphi} J_{m+1}(k \rho \sin \theta)-e^{-i \varphi} J_{m-1}(k \rho \sin \theta)\right] \cos \theta \\
\frac{1}{2}\left[e^{i \varphi} J_{m+1}(k \rho \sin \theta)+e^{-i \varphi} J_{m-1}(k \rho \sin \theta)\right] \cos \theta \\
-J_{m}(k \rho \sin \theta) \sin \theta
\end{array}\right] \\
& \times q(\theta) \mathrm{d} \theta .
\end{aligned}
$$

It can be seen from (17) that at $m=0$ the maximal contribution to the total intensity on the optical axis is coming from the longitudinal component; however, the focal spot size is also affected by the transverse components which contribute to the near-axis region (Table 1 , row 1 ).

The use of a narrow annular diaphragm $[1,2]$ of the radius close to that of the focusing lens and the relative width $\delta$ (normalized with respect to the aperture radius) minimizes the contribution of the transverse components, with the focal region intensity being defined only by the longitudinal component [7] as follows:

$$
\left|E_{0}^{z}(\rho, \varphi, z=0)\right|^{2} \sim \delta^{3 / 2} J_{0}^{2}(k \rho),
$$

whence it follows that the central focal spot radius is $\rho_{\delta} \approx$ $0,38 \lambda$, which corresponds to the diffraction-limited size in
(4). Its intensity is very low because the relative width of the annular aperture is $\delta \ll 1$ (Table 1 , row 2 ).

Introducing a phase jump of $\pi$ radian [19] along the middle radius of the narrow annular aperture (which is equivalent to the destructive interference of two beams)

$$
\tau(\theta)= \begin{cases}0, & \theta<\Theta-\delta, \\ 1, & \Theta-\delta<\theta<\Theta-\frac{\delta}{2}, \\ \exp (i \pi), & \theta \geq \Theta-\frac{\delta}{2},\end{cases}
$$

yields the intensity distribution generated by the (major) longitudinal component in the focal plane $(\mathrm{NA}=1)$ as follows:

$$
\begin{aligned}
& E_{0}^{z}(\rho, \varphi, z=0) \\
& \quad=i k f \int_{0}^{\Theta} \tau(\theta) J_{m}(k \rho \sin \theta) \sin ^{2} \theta \sqrt{\cos \theta} \mathrm{d} \theta \\
& =i k f \int_{0}^{\sin \Theta} \tau(x) x^{2}\left(1-x^{2}\right)^{-1 / 4} J_{0}(k \rho x) \mathrm{d} x \\
& \approx 2^{1 / 4} \delta^{3 / 4}\left[3^{-1 / 4} J_{0}\left(k \rho\left(1-\frac{3 \delta}{4}\right)\right)-J_{0}\left(k \rho\left(1-\frac{\delta}{4}\right)\right)\right] .
\end{aligned}
$$

From (20), it is seen that there is a nonzero intensity region at the focal spot center. The radius of the lowintensity light spot can be estimated using the Bessel function approximation as follows:

$$
\rho_{\delta p h} \approx \frac{\lambda}{\pi} \sqrt{\frac{0.288+0.72 \delta}{0.288+1.488 \delta}} .
$$

By decreasing the annular diaphragm width, we can get a focal spot size smaller than (4): $\rho_{\delta p h} \underset{\delta \rightarrow 0}{\rightarrow}=\lambda / \pi \approx 0.318 \lambda$. Note that (21) predicts an even smaller size for a larger diaphragm width; however, in this case the approximation used to derive the expression becomes invalid. Table 1 (row 3 ) shows the results of focusing with use of the annular diaphragms of width $\delta=0,2$ and a phase $\pi$-jump introduced along the diaphragm's medium radius. The focal spot size is seen to become smaller, FWHM $=0.329 \lambda$, and, though the side lobes become higher, their intensity is smaller than $30 \%$ of the major peak. Such side lobes can be efficiently filtered out $[20,21]$. We also note that for the side-lobe intensity which is lower than a threshold, their influence can be eliminated due to nonlinear interaction of light with the medium [22].

With a full-aperture transmission function with the power dependence on the radial coordinate, the longitudinal component takes the following form:

$$
\begin{aligned}
& E_{0}^{z}(\rho, \varphi, z=0) \\
& \quad=i k f \int_{0}^{\pi / 2}(\sin \theta)^{s+2} J_{0}(k \rho \sin \theta)(\cos \theta)^{1 / 2} \mathrm{~d} \theta .
\end{aligned}
$$


TABLE 1: Results of numerical simulation for a radially polarized beam without vortex components $(m=0)$.

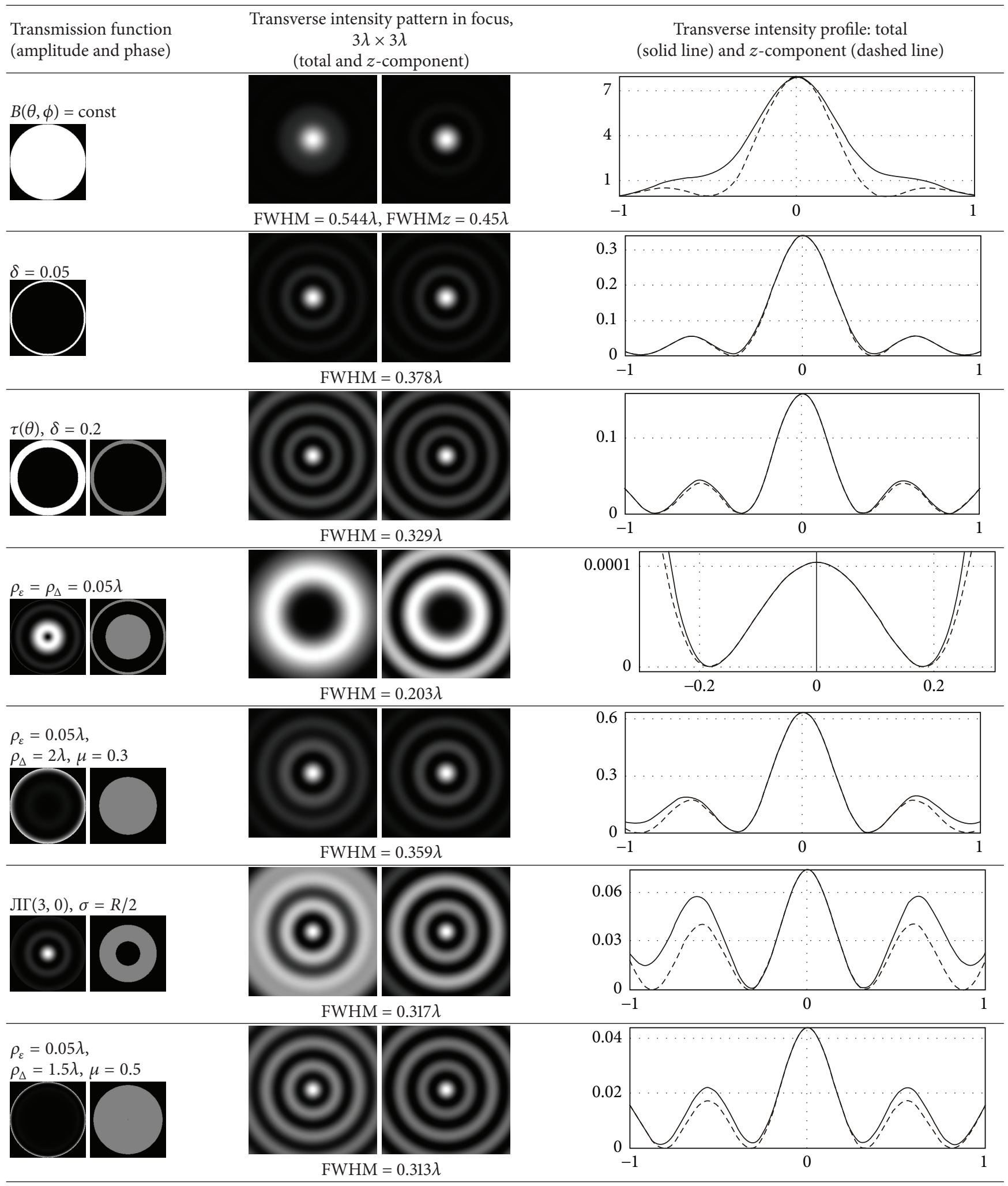


Then, the near-axis intensity is approximately given by [7]

$$
\begin{aligned}
& I_{0}^{z}(\rho, \varphi, z=0) \\
& \approx\left\{\frac{k f}{2} \frac{\Gamma(3 / 4) \Gamma((s+3) / 2)}{\Gamma((s+3) / 2+3 / 4)}\left[1-\frac{(s+3)}{(s+3)+3 / 2}\left(\frac{k \rho}{2}\right)^{2}\right]\right\}^{2},
\end{aligned}
$$

whence it follows that the central focal spot radius is determined by the power $s$ as follows:

$$
\rho_{s}=\frac{\lambda}{\pi} \sqrt{1+\frac{3}{2(s+3)}} .
$$

With increasing $s$, the central light spot radius will tend to $\rho_{s} \rightarrow \infty$

Thus, with use of the full-aperture power function, a tighter focal spot than that with a narrow annular diaphragm $\left(\rho_{\delta} \approx 0.38 \lambda\right)$ can be obtained, so that the resulting focal spot is of practically the same size as that obtained with the annular phase jump $\left(\rho_{\delta p h} \approx 0.38 \lambda\right)$.

However, such an effect is achieved when the power $s$ tends to infinity, actually resulting in the concentration of the incident beam energy on a peripheral lens portion. In this way, the power function serves as an approximation of the narrow annular diaphragm.

Below, a different approach to obtaining a tighter focal spot based on the destructive interference is analyzed. In this case, the coefficients in the superposition in (14) should be selected in a special way [3].

At $m=0$ and in view of (14), (17) in the focal plane takes the following form:

$$
\mathbf{E}_{0}(\rho, \varphi, z=0)=k f\left[\begin{array}{c}
{\left[\frac{\Gamma(1 / 4)}{16}\right] k \rho \cos \varphi \sum_{s=0}^{S} c_{s} \Phi_{s}^{x}(k \rho)} \\
{\left[\frac{\Gamma(1 / 4)}{16}\right] k \rho \sin \varphi \sum_{s=0}^{S} c_{s} \Phi_{s}^{y}(k \rho)} \\
i\left[\frac{\Gamma(3 / 4)}{2}\right] \sum_{s=0}^{S} c_{s} \Phi_{s}^{z}(k \rho)
\end{array}\right],
$$

where

$$
\begin{aligned}
\Phi_{s}^{x}(t) & =\Phi_{s}^{y}(t) \\
& =\frac{\Gamma((s+3) / 2)}{\Gamma((2 s+11) / 4)}{ }_{1} F_{2}\left(\frac{s+3}{2} ; \frac{2 s+11}{4}, 2 ;-\frac{t^{2}}{4}\right), \\
\Phi_{s}^{z}(t) & =\frac{\Gamma((s+3) / 2)}{\Gamma((2 s+9) / 4)}{ }_{1} F_{2}\left(\frac{s+3}{2} ; \frac{2 s+9}{4}, 1 ;-\frac{t^{2}}{4}\right) .
\end{aligned}
$$

Expanding (25) in a power series of $s$ for several initial terms, say, for the transverse components

$$
\begin{aligned}
& E_{0}^{x, y}(\rho, \varphi, z=0) \\
& =k^{2} \rho f \frac{\Gamma(1 / 4)}{16}\left[\begin{array}{c}
\cos \varphi \\
\sin \varphi
\end{array}\right] \\
& \quad \times\left\{\frac{c_{0} \Gamma(3 / 2)}{\Gamma(11 / 4)}{ }_{1} F_{2}\left(\frac{3}{2} ; \frac{11}{4}, 2 ;-\frac{k^{2} \rho^{2}}{4}\right)\right. \\
& \quad+\frac{c_{1} \Gamma(2)}{\Gamma(13 / 4)}{ }_{1} F_{2}\left(2 ; \frac{13}{4}, 2 ;-\frac{k^{2} \rho^{2}}{4}\right) \\
& \quad+\frac{c_{2} \Gamma(5 / 2)}{\Gamma(15 / 4)}{ }_{1} F_{2}\left(\frac{5}{2} ; \frac{15}{4}, 2 ;-\frac{k^{2} \rho^{2}}{4}\right) \\
& \left.\quad+\frac{c_{3} \Gamma(3)}{\Gamma(17 / 4)}{ }_{1} F_{2}\left(3 ; \frac{17}{4}, 2 ;-\frac{k^{2} \rho^{2}}{4}\right)+\cdots\right\},
\end{aligned}
$$

it becomes evident that there is a similar dependence for the same-parity terms. In this case, the Zernike polynomials will be described by simpler relationships [7].

With only zero- and second-power terms of the radial polynomial retained, we obtain

$$
\begin{aligned}
E_{0}^{x, y}(\rho \longrightarrow 0, \varphi, z=0) & \\
\approx & k^{2} f \rho\left[\begin{array}{c}
\cos \varphi \\
\sin \varphi
\end{array}\right] \frac{c_{0} \Gamma(1 / 4)}{16} \frac{\Gamma(3 / 2)}{\Gamma(11 / 4)} \\
\times & \left\{\left(1+\frac{6}{11} \frac{c_{2}}{c_{0}}\right)-\frac{3}{22}\left(1+\frac{2}{3} \frac{c_{2}}{c_{0}}\right)\left(\frac{k^{2} \rho^{2}}{2}\right)\right. \\
& \left.+\frac{1}{66}\left(1+\frac{14}{19} \frac{c_{2}}{c_{0}}\right)\left(\frac{k^{2} \rho^{2}}{2}\right)^{2}\right\}, \\
E_{z}^{0}(\rho \longrightarrow & 0, \varphi, z=0) \\
\approx i k f \frac{c_{0} \Gamma(3 / 4)}{2} \frac{\Gamma(3 / 2)}{\Gamma(9 / 4)} & \times\left\{\left(1+\frac{2 c_{2}}{3 c_{0}}\right)-\frac{1}{3}\left(1+\frac{15 \cdot 2 c_{2}}{13 \cdot 3 c_{0}}\right)\left(\frac{k^{2} \rho^{2}}{2}\right)\right. \\
& \left.+\frac{5}{13 \cdot 24}\left(1+\frac{21 \cdot 2 c_{2}}{17 \cdot 3 c_{0}}\right)\left(\frac{k^{2} \rho^{2}}{2}\right)^{2}\right\} .
\end{aligned}
$$

From (28), it is seen that the contribution of the longitudinal component to the focus $(\rho=0)$ is zero at $c_{2}=-3 c_{0} / 2$. Meanwhile, the contribution of the transverse components is zero at $c_{2}=-11 c_{0} / 6$. Thus, at $c_{2}=-2 c_{0}$, the Zernike polynomial $(2,0)$ provides near-optimal maximization of the longitudinal component contribution [7].

With the analytical computations becoming too cumbersome for a larger number of terms, it appears more convenient to conduct the optimization of the coefficients using a numerical procedure under certain conditions.

In particular, the focal spot can be arbitrarily decreased in size based on a manyfold growth of the side lobes, with 


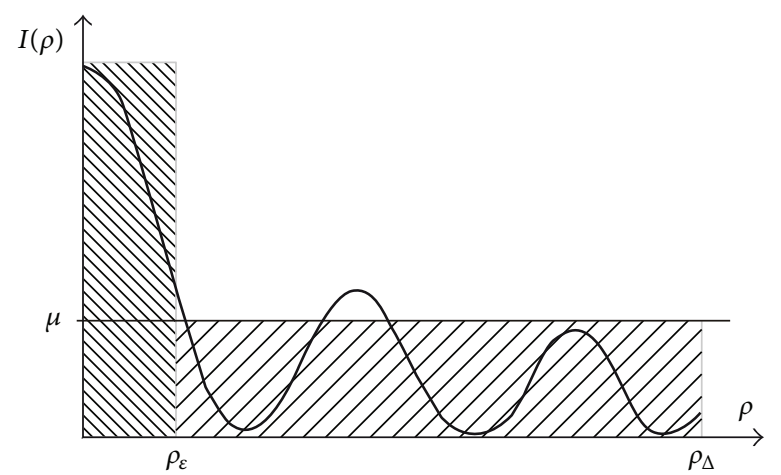

Figure 2: Illustration of the target function in the focal plane at a fixed value of the polar angle $\varphi$.

practically the entire energy being redirected from the focus $[23,24]$ (Table 1 , row 4). To achieve a trade-off between a smaller focal spot and the amount of light energy coming to the focus, we impose a condition that the energy should be concentrated in the focal plane within a circle of radius $\rho_{\varepsilon}$ and a desired level $\mu$ of side lobes' intensity is provided within a ring with radii $\left[\rho_{\varepsilon}, \rho_{\Delta}\right]$ (Figure 2 ).

The corresponding functional (16) was minimized by fitting the coefficients in (14) using Brent's method [12]. Note that beyond the radius $\rho_{\Delta}$ the intensity level is not controlled and can be essentially higher than that at the center. Alongside the energy loss, an increase in the region under control usually results in a larger focal spot.

Row 5 of Table 1 shows the focusing results for the full-aperture transmission function described by a thirdorder polynomial whose coefficients were chosen so as to concentrate the energy in a circle of radius $\rho_{\varepsilon}=0.05 \lambda$, while keeping the side-lobe intensity in the ring of radii $[0.05 \lambda, 2 \lambda]$ not higher than $\mu=0.3$ of the focal spot intensity. In this case, the focal spot is smaller in size when compared with a focal spot obtained with a narrow annular aperture, whereas the focal intensity in the former case is two times higher.

In [6], it was proposed that the focal spot should be made smaller with the aid of the transmission function proportional to the Laguerre-Gauss (LG) modes. In this case, the focusing results depend on the waist radius and the LG mode radial number. Note that the use of a higher-order LG mode does not have to lead to a smaller focal spot. In particular, for $\sigma=R / 2$, the focusing results were the following: $\mathrm{FWHM}=0.437 \lambda$ (for the $\mathrm{LG}$ mode radial number $n=1)$, FWHM $=0.875 \lambda(n=2)$, FWHM $=0.317 \lambda$ $(n=3$, see Table 1 , row 6$), \mathrm{FWHM}=0.384 \lambda(n=4)$, and $\mathrm{FWHM}=0.475 \lambda(n=5)$.

In this case, essential growth of the side lobes is also observed, with their intensity approaching $\mu=0.86$ of the total maximum (Table 1 , row 6).

With the LG modes, the result can be improved with the side-lobe level attaining $\mu=0.5$ and the focal spot size remaining unchanged $(\mathrm{FWHM}=0.313 \lambda$ ) by applying the optimization algorithm to the superposition of (14); see Table 1, row 7.
5.2. Minimizing the Light Spot Radius. If the radially polarized incident wave contains first-order vortex components $(|m|=1)$, the transverse components will take nonzero values on the optical axis, whereas the longitudinal component, on the contrary, will form an annular distribution pattern [7]. Note that the contribution of the longitudinal component into the total intensity is comparable with that of the transverse components. It is most essential that this factor should be taken into consideration when dealing with the interaction of light with the media being selectively sensitive to the different electric field components [25].

The longitudinal wave component generates a distribution given by

$$
\begin{aligned}
E_{z}(\rho, \varphi, z=0) \\
=-k f \exp (i \varphi) \\
\quad \times \int_{0}^{\pi / 2} \sum_{s=0}^{S} c_{s} \cdot(\sin \theta)^{s+2} J_{1}(k \rho \sin \theta)(\cos \theta)^{1 / 2} \mathrm{~d} \theta .
\end{aligned}
$$

For the even-valued powers of sine $(s=2 l)$, we will use the formula [26]

$$
\begin{aligned}
\int_{0}^{1} x^{2 l+2} & \left(1-x^{2}\right)^{-1 / 4} J_{1}(k \rho x) \mathrm{d} x \\
= & \frac{1}{2}(2)_{l} \Gamma\left(\frac{3}{4}\right)\left(\frac{2}{k \rho}\right)^{l+3 / 4} \\
& \times \sum_{k=0}^{l} \frac{(-1)^{k}}{(2)_{k}}\left(\begin{array}{l}
l \\
k
\end{array}\right)\left(\frac{k \rho}{2}\right)^{k} J_{k+l+7 / 4}(k \rho),
\end{aligned}
$$

whereas for the odd-valued powers $(s=2 l+1)$, a more general formula needs to be used [26] as follows:

$$
\begin{aligned}
\int_{0}^{1} x^{s+1}\left(1-x^{2}\right)^{-1 / 4} J_{1}(k \rho x) \mathrm{d} x \\
=\frac{k \rho}{4} \frac{\Gamma(3 / 4) \Gamma((s+3) / 2)}{\Gamma(s / 2+9 / 4)} \\
\quad \times{ }_{1} F_{2}\left(\frac{s+3}{2} ; \frac{s}{2}+\frac{9}{4}, 2 ;-\frac{(k \rho)^{2}}{4}\right) .
\end{aligned}
$$

With no transmission functions whatsoever (which corresponds to $l=0$ ), we have the following:

$$
\int_{0}^{1} x^{2}\left(1-x^{2}\right)^{-1 / 4} J_{1}(k \rho x) \mathrm{d} x=\frac{1}{2} \Gamma\left(\frac{3}{4}\right)\left(\frac{2}{k \rho}\right)^{3 / 4} J_{7 / 4}(k \rho) .
$$

If the Bessel function at small argument values is approximated by

$$
J_{\nu}(x \longrightarrow 0) \approx \frac{1}{\Gamma(\nu+1)}\left(\frac{x}{2}\right)^{\nu}\left[1-\frac{x^{2}}{4(\nu+1)}\right],
$$


the right-hand side of (32) in the optical axis vicinity can be estimated as $(4 k \rho / 21)\left(1-\left((k \rho)^{2} / 11\right)\right)$. The shadow spot's radius can be derived by setting the derivative with respect to $\rho$ equal to zero as follows:

$$
\rho_{0 d}=\frac{\lambda}{2 \pi} \sqrt{\frac{11}{3}} \approx 0.305 \lambda .
$$

For the odd-valued powers $s$, it is also possible to estimate the right-hand side of (31). For the near-axis region, we can write down

$$
{ }_{1} F_{2}\left(\frac{s+3}{2} ; \frac{2 s+9}{4}, 2 ;-\frac{(k \rho)^{2}}{4}\right) \approx 1-\frac{s+3}{2 s+9} \cdot \frac{(k \rho)^{2}}{4}
$$

and deduce the shadow spot's radius as follows:

$$
\rho_{s} \approx \frac{\lambda}{\pi} \sqrt{\frac{2 s+9}{3 s+9}}
$$

It can be seen from relation (36) that as the value of $s$ increases, the shadow spot's radius decreases, tending to the diffraction-limited value $\rho_{s} \underset{s \rightarrow \infty}{\rightarrow} \sqrt{2 / 3}(\lambda / \pi) \approx 0.26 \lambda$. However, based on the superposition in (14), a better result can be achieved for the polynomials at lower-order $s$ due to mutual interference.

With a narrow annular diaphragm, (29) takes a simple form independent of the radial polynomials as follows:

$$
\sum_{s=0}^{S} c_{s} \int_{1-\delta}^{1} x^{s+2}\left(1-x^{2}\right)^{-1 / 4} J_{1}(k \rho x) \mathrm{d} x \approx \frac{4 \delta^{3 / 4}}{3 \cdot(2)^{1 / 4}} J_{1}(k \rho) .
$$

In this case, the shadow spot's radius is $\rho_{\delta d} \approx 0.29 \lambda$.

Thus, the spot is smaller in size than that obtained with a conventional full-aperture focusing system but larger than that achieved based on the use of the radial polynomial.

Table 2 gives the numerically simulated results for the radially polarized beam containing a first-order phase vortex. In this case, the parameter FWHM describes the shadow spot size as a full-width half-maximum intensity at the light barrier's inner walls.

Table 2 suggests that the presence of the transverse components is a hindrance to obtaining a "pure" result in the total intensity (Table 2 , row 1 ).

When focusing light with the aid of a narrow annular diaphragm, the minimal light ring is achieved with the transverse components' contribution being minimal (Table 2, row 2). However, an essential energy loss is observed.

Based on the polynomials, a trade-off can be achieved between the decrease of the light ring's size and the energy loss in the central part: row 3 of Table 2 shows the result for a near-minimal ring with the 2.5 times intensity.

As a rule, a tighter focal spot is achieved by redistributing the energy to the peripheral lens part (approximation of a narrow annular slit) or destructive interference between the beams generated by different lens regions (similarly to a diaphragm with a phase jump, (19)). In the former case, obtaining a still narrower slit requires increasing the polynomial order. In the latter case, the lens pupil can be divided into radial zones, with low-order polynomials being considered for each zone.

The optimization results for a three-zone pupil function-a small-radius central (shielding) zone and two zones containing third-order polynomials-are shown in Table 2, row 4 . In this case, the focal spot is being tightened more effectively but, as in the previous case, at the expense of higher side lobes, which were kept at a level of no higher than $\mu=0.9$ on the radius $\rho_{\Delta}=1.2 \lambda$. Note that, for the shadow focal spot, the side lobes' effect is of lower significance when compared with the light spot because it is the region found within the light barrier which serves the useful purpose.

\section{Azimuthal Polarization}

For azimuthal polarization, the field in the focal plane $(z=0)$ takes the followimg form:

$$
\begin{aligned}
& \mathbf{E}_{m}(\rho, \varphi, z) \\
& =-i^{m+1} k f \exp (i m \varphi) \\
& \times \int_{0}^{\Theta}\left[\begin{array}{c}
\frac{1}{2}\left[e^{i \varphi} J_{m+1}(k \rho \sin \theta)+e^{-i \varphi} J_{m-1}(k \rho \sin \theta)\right] \\
\frac{-i}{2}\left[e^{i \varphi} J_{m+1}(k \rho \sin \theta)-e^{-i \varphi} J_{m-1}(k \rho \sin \theta)\right] \\
0
\end{array}\right] \\
& \times q(\theta) \mathrm{d} \theta,
\end{aligned}
$$

whence it follows that the $z$-component is zero at any $m$.

Upon sharp focusing, the electric field on the optical axis $(\rho=0)$ will contain nonzero components just for one singularity order, $|m|=1$. For all the rest values, $|m| \neq 1$, an absolute intensity zero will be formed at the axis points, because all components will be equal to zero. Because of this, the azimuthal polarization is best suited for generating a shadow spot (Table 3 , row 1 ).

At $m=0$ and in view of (14), (38) is reduced to a compact form as follows:

$$
\begin{aligned}
& \mathbf{E}_{0}(\rho, \varphi, z=0) \\
& =k f\left[\begin{array}{c}
\sin \varphi \\
-\cos \varphi \\
0
\end{array}\right] \int_{0}^{\pi / 2} \sum_{s=0}^{S} c_{s} \cdot(\sin \theta)^{s+1} \\
& \quad \times J_{1}(k \rho \sin \theta)(\cos \theta)^{1 / 2} \mathrm{~d} \theta .
\end{aligned}
$$

The integrals in (39) can be taken with the aid of hypergeometric functions whose analysis [7] has shown that, while depending on the power $s$, the radius of the resulting light ring is limited by $\rho_{\min } \approx 0.26 \lambda$ (lower estimate). 
TABLE 2: Results of numerical simulation for the radially polarized beam with a first-order phase vortex $(m=1)$.

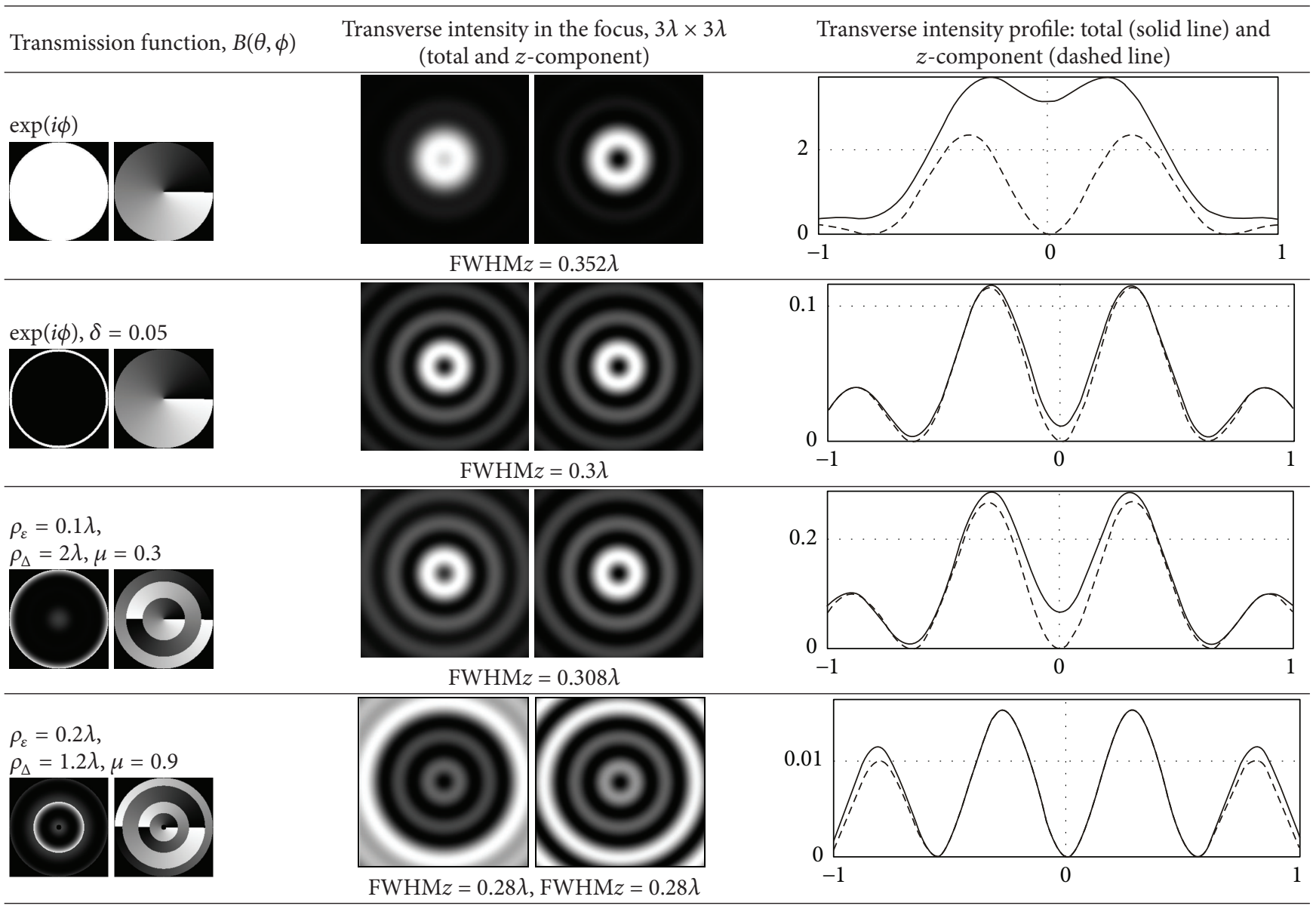

After introducing a narrow annular diaphragm, the integral in (39) takes a simple, polynomial-independent form as follows:

$$
\int_{\pi / 2-\delta}^{\pi / 2} J_{1}(k \rho \sin \theta) \sin \theta(\cos \theta)^{1 / 2} \mathrm{~d} \theta \sim \delta^{3 / 4} J_{1}(k \rho) ;
$$

that is, the light ring's radius is determined by the first maximum of the first-order Bessel function $\rho_{\delta} \approx 0.293 \lambda$, which corresponds to the scalar limit in (10) (Table 3, row 2). The intensity of the focal ring drops with a decrease in the annular diaphragm width $\delta$.

Also note that introducing an additional $\pi$-jump of phase along the medium radius of the diaphragm enables obtaining a smaller focal spot of FWHM $=0.329 \lambda$ at the penalty of further reduction of the ring intensity (Table 3, row 3 ).

Based on the superposition of (14), an attempt can be made to find a trade-off between attaining a smaller focal ring and the energy loss at the center. In particular, this can be implemented by keeping the side-lobe intensity relatively low when compared with the central focal ring intensity for a comparatively wide region (Table 3 , row 4 ).

By allowing the side-lobe intensity to reach that of the central ring (Table 3, row 5), it becomes possible to break the diffraction limit, obtaining the central ring intensity 2 times the intensity obtained with a narrow annular diaphragm.

The central focal spot can be made still smaller only at the penalty of the considerable energy loss (Table 3, row 6).

\section{Circular Polarization}

The circularly polarized beams are attractive because they can be easily obtained from linearly polarized light emitted by the majority of laser sources. Unlike the previously discussed radially and azimuthally polarized beams, no complex or expensive devices are required to obtain them.

For a circularly polarized beam, there is a correlation between the polarization sign and the vortex order sign. If the vortex order $|m| \leq 2$ has the opposite sign to the polarization sign, there will be nonzero components on the optical axis $(\rho=0)$. This factor will have an adverse effect on an attempt to obtain a circular distribution of the total intensity.

For certainty, “+”-sign circular polarization is analyzed below. Then, at $m=0$ and $m=-2$, the transverse components will make a nonzero contribution to the intensity on the optical axis, whereas the longitudinal component will form a diffraction ring. We note that at $m=-2$ the transverse components' energy on the axis is lower than it is at $m=0$. 
TABLE 3: Results of numerical simulation for the azimuthally polarized beam without vortex components $(m=0)$.

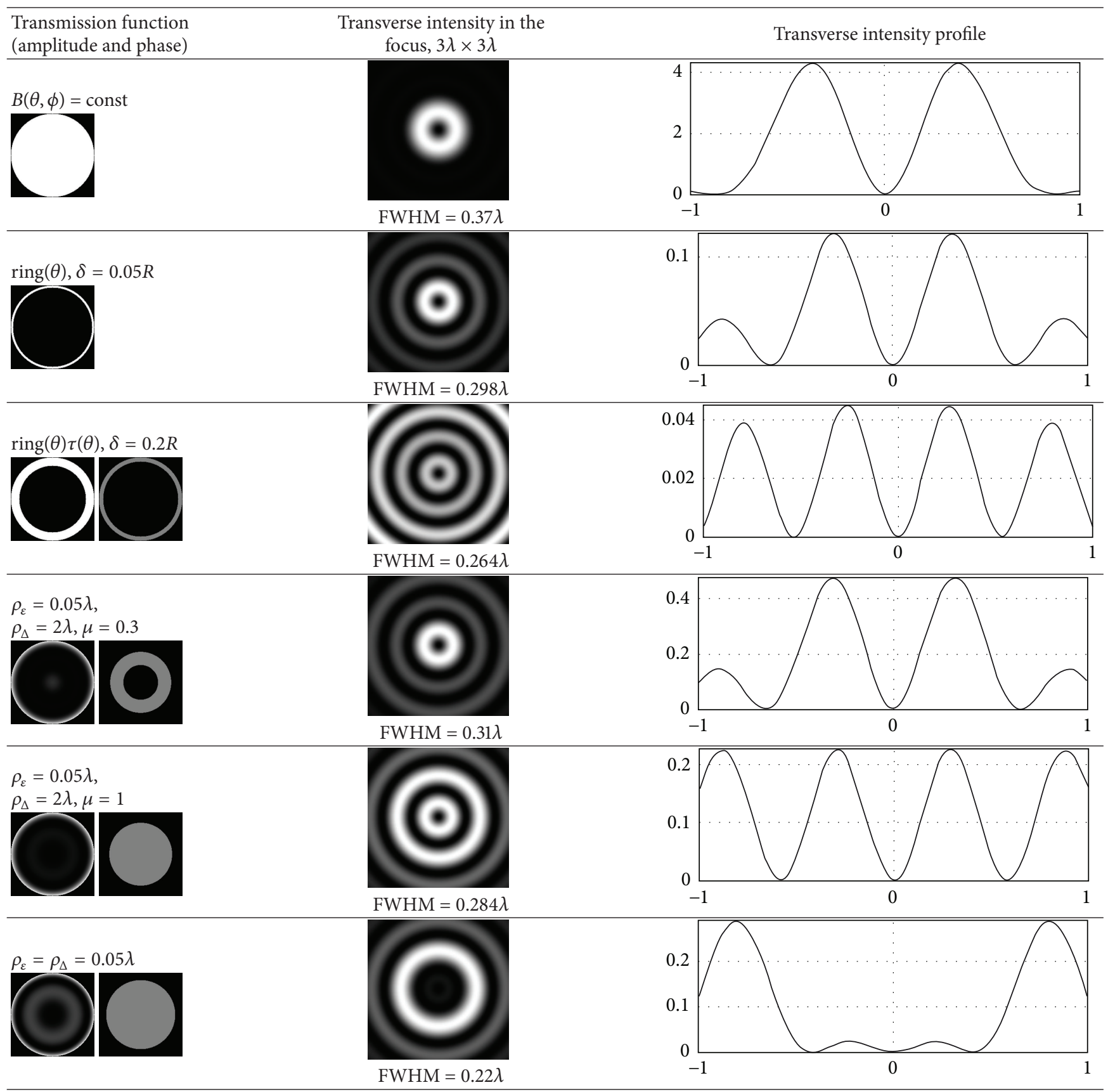

At $m=-1$, the situation is the opposite, but for the high NA focusing systems, the contribution from the longitudinal components is normally large. Because of this, for a shadow spot to be formed, the variant with $m=-2$ is better-suited. Then, the field in the focal region takes the following form:

$$
\begin{aligned}
& \mathbf{E}(\rho, \varphi, z=0) \\
& =\frac{-k f}{2} \\
& \quad \times \int_{0}^{\pi / 2} \sum_{s=0}^{S} c_{s} \cdot(\sin \theta)^{s+1}
\end{aligned}
$$

$$
\times\left[\begin{array}{c}
e^{-i 2 \varphi} J_{-2}(t)+\frac{1}{2}\left[e^{-i 2 \varphi} J_{-2}(t)-J_{0}(t)\right](\cos \theta-1) \\
i\left\{e^{-i 2 \varphi} J_{-2}(t)+\frac{1}{2}\left[e^{-i 2 \varphi} J_{-2}(t)+J_{0}(t)\right](\cos \theta-1)\right\} \\
-i e^{-i \varphi} J_{-1}(t) \sin \theta
\end{array}\right]
$$$$
\times(\cos \theta)^{1 / 2} \mathrm{~d} \theta
$$

where $t=k \rho \sin \theta$.

With a narrow annular diaphragm employed for the longitudinal component, the result will be the same as in 
TABLE 4: Results of numerical simulation for the circular "+"-polarization in the presence of a second-order optical vortex $(m=-2)$.

Transmission function, $B(\theta, \phi)$
Transverse intensity in the focus, $3 \lambda \times 3 \lambda$
total and $z$-component)

(36). However, the contribution of the nonzero longitudinal components in the focus also becomes significant. As a result, in terms of the total intensity, the ring becomes fuzzier in structure than in the case of the full NA system, as seen from the comparison of row 1 and row 2 of Table 4 . The use of the optimization procedure improves the situation just insignificantly when compared with the situation when no additional apodization of the focusing system's output pupil is used (Table 4, row 3).

Thus, an attempt to obtain a clearly observed light ring in terms of the total intensity has proved to be unsuccessful. Because of this, we analyze a circularly polarized "+"-wave with the first-order optical vortex of the same sign as follows:

$$
\begin{aligned}
& \mathbf{E}(\rho, \varphi, z=0) \\
& =\frac{i k f}{2} \\
& \quad \times \int_{0}^{\pi / 2} \sum_{s=0}^{S} c_{s} \cdot(\sin \theta)^{s+1} \\
& \quad \times\left[\begin{array}{c}
e^{i \varphi} J_{1}(t)+\frac{1}{2}\left[e^{i \varphi} J_{1}(t)-e^{i 3 \varphi} J_{3}(t)\right](\cos \theta-1) \\
i\left\{e^{i \varphi} J_{1}(t)+\frac{1}{2}\left[e^{i \varphi} J_{1}(t)+e^{i 3 \varphi} J_{3}(t)\right](\cos \theta-1)\right\}
\end{array}\right] \\
& \quad \times(\cos \theta)^{1 / 2} \mathrm{~d} \theta, \quad-i e^{i 2 \varphi} J_{2}(t) \sin \theta
\end{aligned}
$$

where $t=k \rho \sin \theta$.
From (42), it is seen that, in the general case, a smaller diffraction ring will be formed for the transverse components in comparison with that formed for the longitudinal component (Table 5). Hence, in this case, the use of a narrow annular diaphragm is inefficient, only leading to the extraction of the longitudinal component (Table 5, row 2).

The use of the superposition of (14), with the coefficients calculated so as to minimize the light ring, enables the light ring size to be decreased in terms of the total intensity (see Table 5, row 3), but the results are not better than those obtained for the azimuthally polarized beam.

Thus, for widely used and easy to realize polarization types (circular or linear), the optimal results can be only achieved for individual electric field components-the longitudinal or transverse ones. In the total intensity, the optimization effect is deteriorated owing to the combined contribution of different components. In this case, the contribution of the undesirable component can be eliminated with the use of materials selectively sensitive to different electric field components [25].

\section{Conclusion}

We have looked into the ways of minimizing the light/shadow focal spot size for differently polarized incident beams under the controlled side-lobe growth by means of an optimal apodization of the focusing system. The apodization that has been proposed involves the introduction of a vortex phase dependence on the angle and the polynomial amplitude dependence on the radius. The optimization of the polynomial coefficients has been performed using Brent's method with the aim of preserving the energy efficiency and keeping the side lobes at desired level. 
TABLE 5: Results of numerical simulation for “+”-polarization in the presence of a first-order optical vortex $(m=1)$.

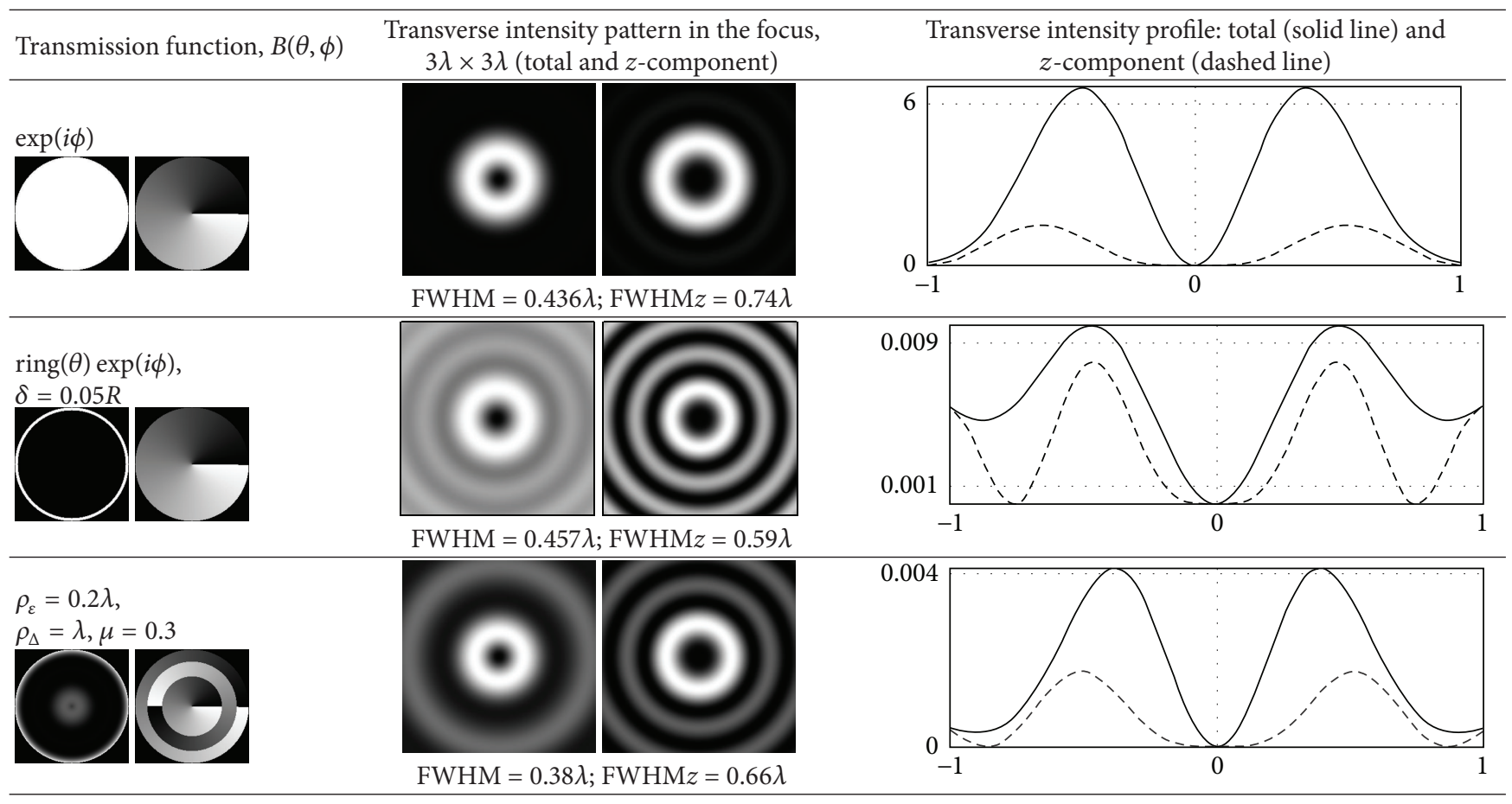

The study has shown that the radial polarization can be efficiently used for generating a tighter focal spot and the azimuthal polarization-for the light ring.

In this case, the diffraction-limited size predicted by the scalar theory is achieved by merely introducing a narrow annular diaphragm shielding off the central part of the focusing system. The efficiency can be enhanced with the use of a wider annular diaphragm with a radial phase jump. With such optical element, it is also possible to obtain a tighter focal spot than that predicted by the scalar theory of diffraction.

The only way to make the light/shadow focal spot essentially smaller is by redirecting the energy to the side lobes. In this case, the growth of the side lobes in a near-focus region can be controlled by means of the amplitude distribution defined by a radial polynomial, which can be optimized, for example, using Brent's method.

The achievement of a trade-off between obtaining a smaller focal spot and retaining the energy in the center depends on the area of the focal region under control. Beyond the region under control, the intensity level can be essentially higher than that in the center. However, alongside the lower energy loss, the expansion of the region under control usually results in a larger focal spot. Thus, the results obtained are in full agreement with the theory of Toraldo di Francia.

For widely used and easy to realize polarization types, in particular, circularly and linearly polarized beams, analogous results can be only achieved for individual electric field components-the longitudinal or transverse ones. In the total intensity, the optimization effect is deteriorated owing to the combined contribution of different components. In this case, the contribution of the undesirable component can be eliminated with the use of materials selectively sensitive to different electric field components.

\section{Acknowledgments}

The work was financially supported by the Russian Foundation for Basic Research (Grant 13-07-00266) and by the Ministry of Education and Science of Russian Federation.

\section{References}

[1] S. Quabis, R. Dorn, M. Eberler, O. Glöckl, and G. Leuchs, "Focusing light to a tighter spot," Optics Communications, vol. 179, no. 1, pp. 1-7, 2000.

[2] R. Dorn, S. Quabis, and G. Leuchs, "Sharper focus for a radially polarized light beam," Physical Review Letters, vol. 91, no. 23, pp. 2339011-2339014, 2003.

[3] L. E. Helseth, "Smallest focal hole," Optics Communications, vol. 257, no. 1, pp. 1-8, 2006.

[4] S. N. Khonina, N. L. Kazanskiy, and S. G. Volotovsky, "Vortex phase transmission function as a factor to reduce the focal spot of high-aperture focusing system," Journal of Modern Optics, vol. 58, no. 9, pp. 748-760, 2011.

[5] S. N. Khonina and S. G. Volotovsky, "Controlling the contribution of the electric field components to the focus of a highaperture lens using binary phase structures," Journal of the Optical Society of America A, vol. 27, no. 10, pp. 2188-2197, 2010.

[6] Y. Kozawa and S. Sato, "Sharper focal spot formed by higherorder radially polarized laser beams," Journal of the Optical Society of America A, vol. 24, no. 6, pp. 1793-1798, 2007. 
[7] S. N. Khonina, A. V. Ustinov, and E. A. Pelevina, "Analysis of wave aberration influence on reducing focal spot size in a highaperture focusing system," Journal of Optics, vol. 13, no. 9, Article ID 095702, 2011.

[8] J. Arlt and M. J. Padgett, "Generation of a beam with a dark focus surrounded by regions of higher intensity: the optical bottle beam," Optics Letters, vol. 25, no. 4, pp. 191-193, 2000.

[9] S. W. Hell and J. Wichmann, "Breaking the diffraction resolution limit by stimulated emission: stimulated-emissiondepletion fluorescence microscopy," Optics Letters, vol. 19, no. 11, pp. 780-782, 1994.

[10] D. P. Biss, K. S. Youngworth, and T. G. Brown, "Dark-field imaging with cylindrical-vector beams," Applied Optics, vol. 45, no. 3, pp. 470-479, 2006.

[11] S. N. Khonina, N. L. Kazanskiy, and S. G. Volotovsky, "Influence of vortex transmission phase function on intensity distribution in the focal area of high-aperture focusing system," Optical Memory and Neural Networks, vol. 20, no. 1, pp. 23-42, 2011.

[12] R. P. Brent, Algorithms for Minimization without Derivatives, Prentice-Hall, New York, NY, USA, 1973.

[13] M. Abramovitz and I. A. Stegun, Eds., Handbook of Mathematical Functions, Applied Math. Series, National Bureau of Standards, 1965.

[14] S. N. Khonina and I. Golub, "Enlightening darkness to diffraction limit and beyond: comparison and optimization of different po-larizations for dark spot generation," Journal of the Optical Society of America A, vol. 29, no. 7, pp. 1470-1474, 2012.

[15] Q. Zhan, "Cylindrical vector beams: from mathematical concepts to applications," Advances in Optics and Photonics, vol. 1, pp. 1-57, 2009.

[16] S. N. Khonina and I. Golub, "Optimization of focusing of linearly polarized light," Optics Letters, vol. 36, no. 3, pp. 352354, 2011.

[17] B. Richards and E. Wolf, "Electromagnetic diffraction in optical systems. II. Structure of the image field in an aplanatic system," Proceedings of the Royal Society A, vol. 253, pp. 358-379, 1959.

[18] http://alglib.sources.ru/.

[19] S. N. Khonina and A. V. Ustinov, "Sharper focal spot for a radially polarized beam using ring aperture with phase jump," Journal of Engineering, vol. 2013, Article ID 512971, 8 pages, 2013.

[20] T. R. M. Sales and G. M. Morris, "Diffractive superresolution elements," Journal of the Optical Society of America A, vol. 14, no. 7, pp. 1637-1646, 1997.

[21] J. Bewersdorf, A. Egner, and S. W. Hell, "4pi-confocal microscopy is coming of age," G.I.T. Imaging \& Microscopy, vol. 4, pp. 24-25, 2004.

[22] L. E. Helseth, "Breaking the diffraction limit in nonlinear materials," Optics Communications, vol. 256, no. 4-6, pp. 435438, 2005.

[23] G. T. di Francia, "Degrees of freedom of an image," Journal of the Optical Society of America, vol. 59, no. 7, pp. 799-804, 1969.

[24] F. M. Huang and N. I. Zheludev, "Super-resolution without evanescent waves," Nano Letters, vol. 9, no. 3, pp. 1249-1254, 2009.

[25] T. Grosjean and D. Courjon, "Photopolymers as vectorial sensors of the electric field," Optics Express, vol. 14, no. 6, pp. 2203-2210, 2006.

[26] A. P. Prudnikov, A. Yu. Brychkov, and O. I. Marichev, Integrals and Series. Vol. 2. Special Functions, Gordon \& Breach Sci. Publ., New York, NY, USA, 1990. 

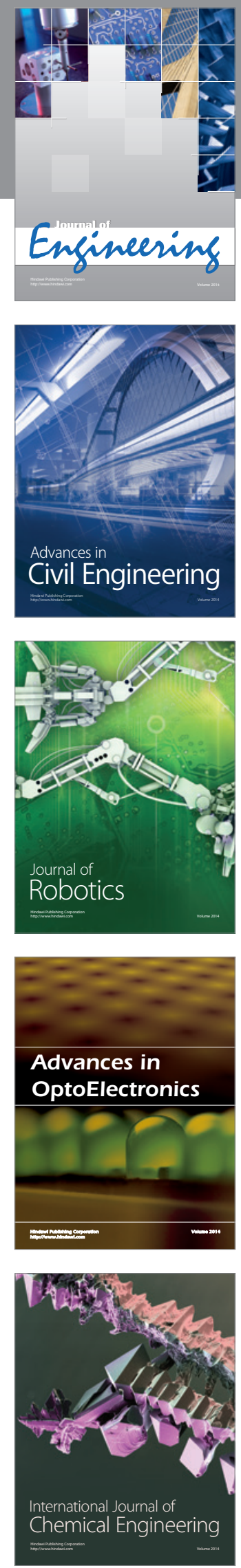

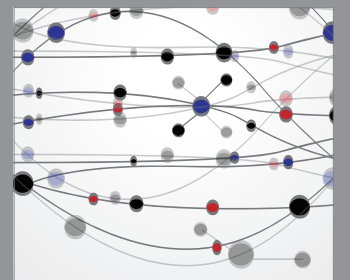

The Scientific World Journal
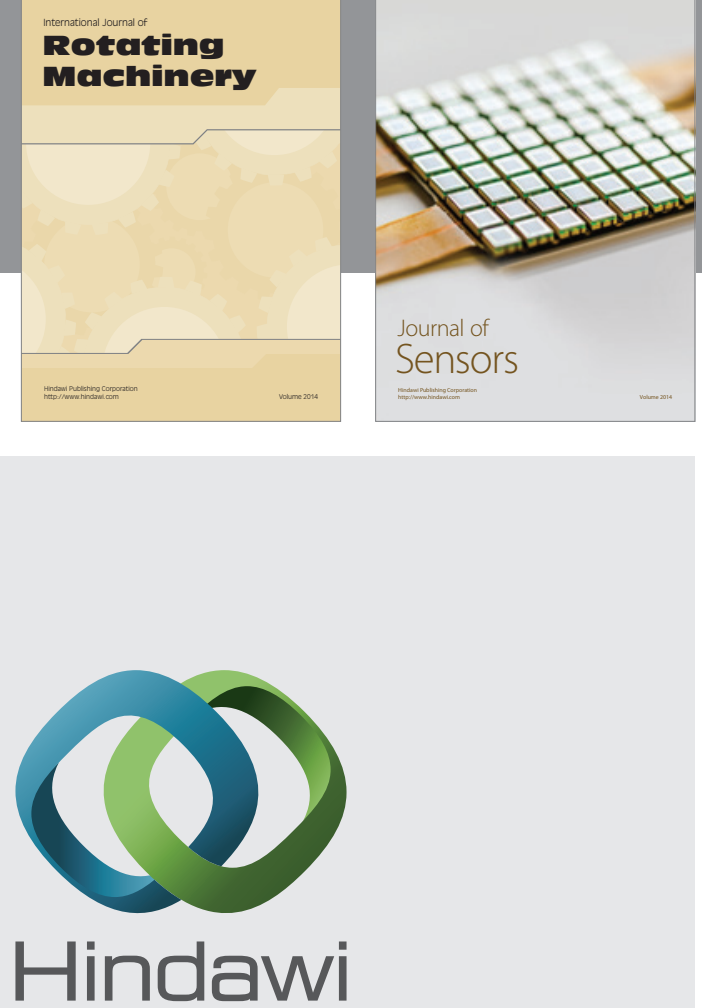

Submit your manuscripts at http://www.hindawi.com
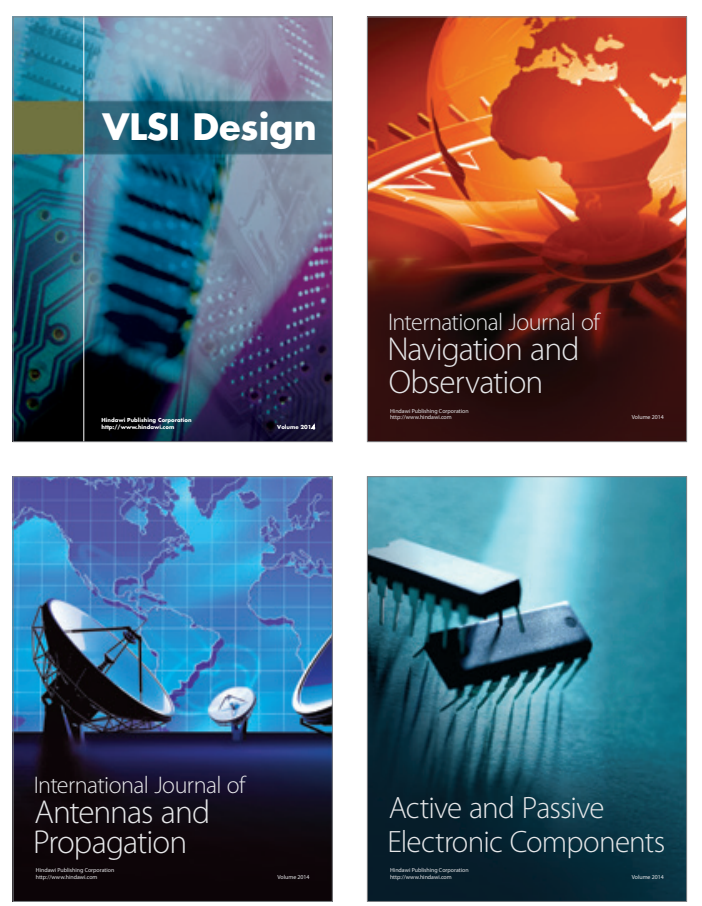
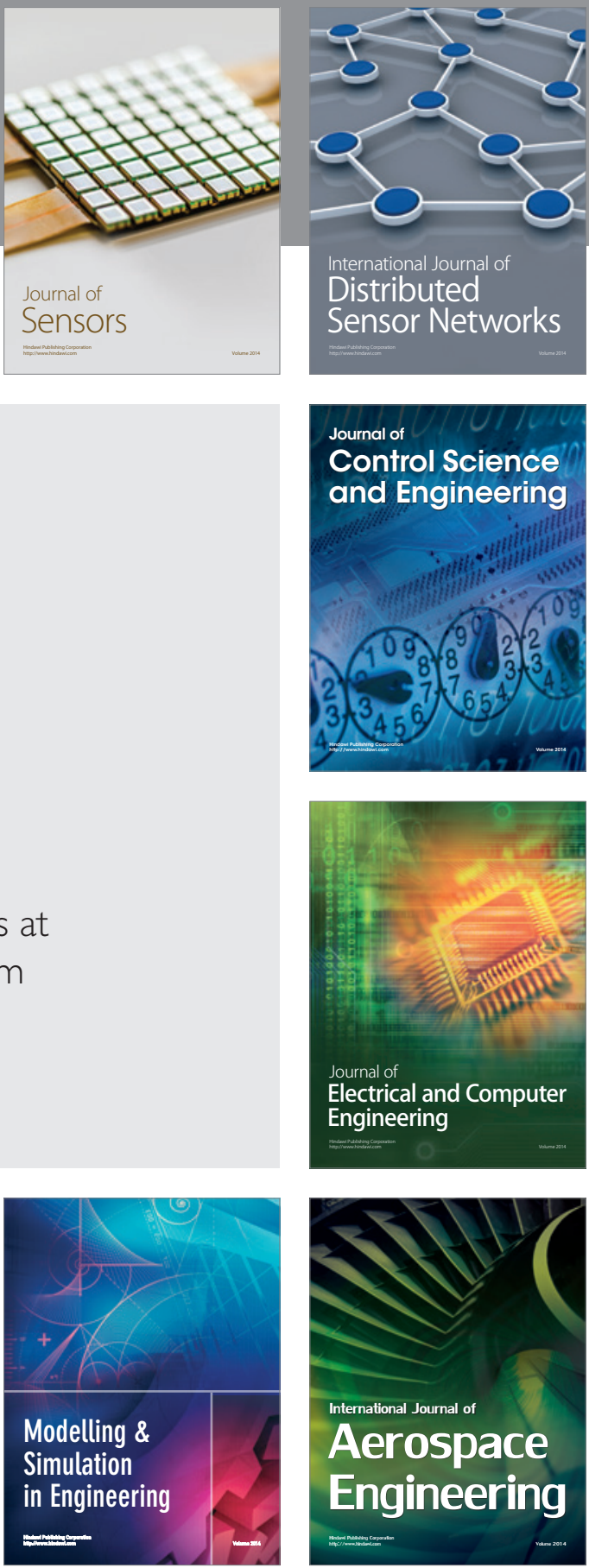

Journal of

Control Science

and Engineering
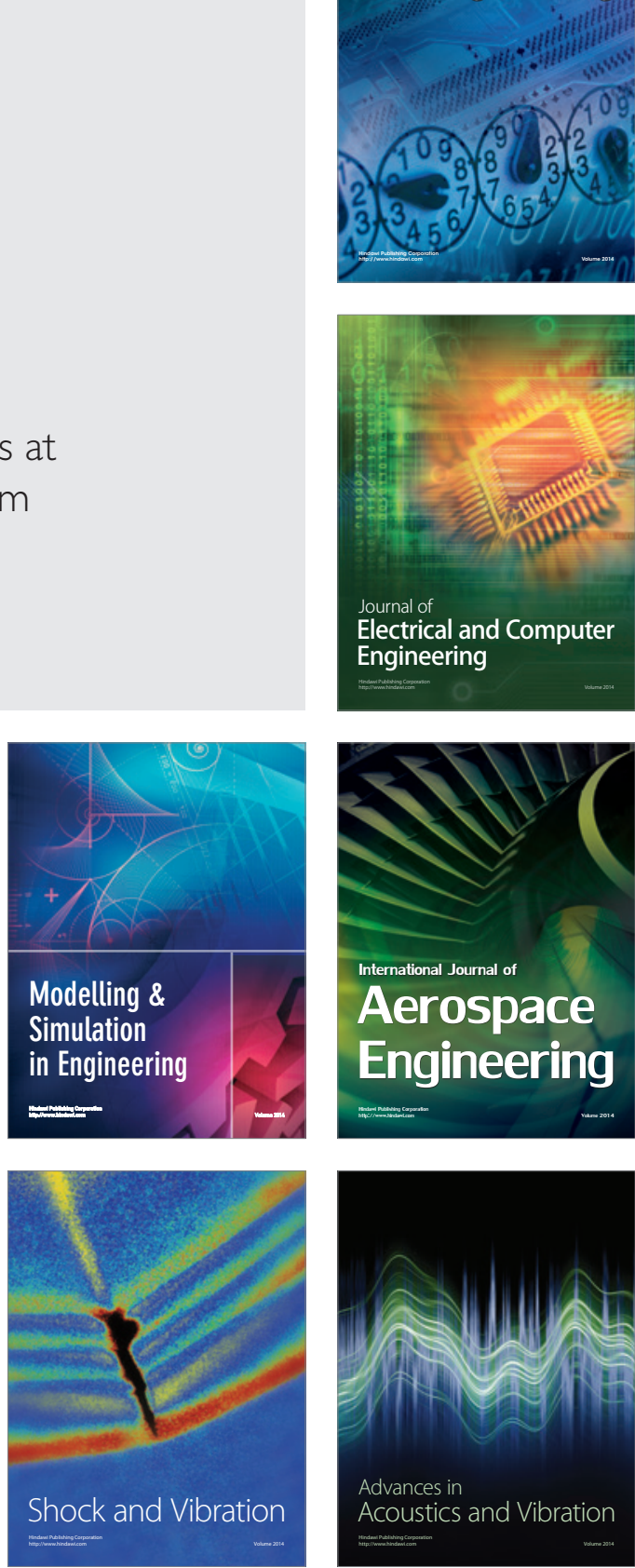